Results: By November 2017, 997 RA-patients were included in Reumanet Bernhoven. $56 \%(n=557)$ used it at least once in this 6 month period. $13 \%(n=70)$ of them used the self monitoring tool. The remaining patients, $44 \%(n=440)$ were questioned for not using Reumanet at home. $55 \%(n=242)$ of these patients responded and $14 \%(n=62)$ do want to participate yet, $26 \%(n=114)$ did not have a computer or email address, $16 \%(n=71)$ did not want to using a digital environment at home.

Conclusions: Half of the RA-patients used Reumanet at home of which $13 \%$ performed self-monitoring of their disease activity in this online personal health environment. Reumanet will be seen as a promising tool to involve patients in their disease management and can be helpful for shared decision making between patient and health professional. In future, more research is needed to optimise patient self-management in a digital health environment in order to increase its usage.

Disclosure of Interest: None declared

DOI: 10.1136/annrheumdis-2018-eular.3618

\section{OP0160-HPR DETERMINANTS OF HAPPINESS AND QUALITY OF LIFE IN PATIENTS WITH RHEUMATOID ARTHRITIS: A STRUCTURAL EQUATION MODELLING APPROACH}

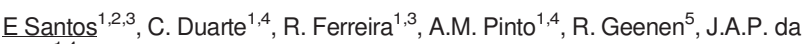
Silva $^{1,4}$, on behalf of 'Promoting happiness through excellence of care'. ${ }^{1}$ Rheumatology department, Centro Hospitalar e Universitário de Coimbra, Coimbra; ${ }^{2}$ Instituto de Ciências Biomédicas Abel Salazar, Porto; ${ }^{3}$ Health Sciences Research Unit: Nursing; ${ }^{4}$ Faculdade de Medicina da Universidade de Coimbra, Coimbra, Portugal; ${ }^{5}$ Psychology, Utrecht University, Utrecht, Netherlands

Background: Remission is the core target of disease management in rheumatoid arthritis (RA), but the ultimate goal of medical care is to improve patients' enjoyment of life, a concept akin to happiness. What is the contribution of disease control towards happiness and what other means may the health professional consider towards that goal?

Objectives: To examine the determinants of happiness and quality of life (QoL) in patients with rheumatoid arthritis (RA), with emphasis on disease activity, disease impact and personality traits.

Methods: This is an ancillary analysis of an observational, cross-sectional study. Consecutive patients were assessed on disease activity, disease impact, personality, QoL and happiness. Structural equation modelling estimation was used to assess the associations between these dimensions, pursuing three hypotheses: $\mathrm{H}_{1}$ - Disease activity and perceived impact of disease are negatively associated to overall QoL and happiness in patients with $\mathrm{RA} ; \mathrm{H}_{2}-$ 'Positive' personality traits are related to happiness both directly and indirectly through perceived disease impact; $\mathrm{H}_{3}-$ Happiness has a mediating effect in the relation between impact of disease and QoL

Results: Data from 213 patients was analysed. Results obtained in the structural equation measurement model indicated a good fit $\left[\chi^{2} / d f=1.38 ; C F l=0.98\right.$; $\mathrm{GFI}=0.92 ; \mathrm{TLI}=0.97 ; \mathrm{RMSEA}=0.04]$ and supported all three driving hypotheses (figure 1). Happiness was positively related to 'positive' personality (total effect of 0.56 , with a direct effect of $\beta=0.50, p<0.001$ and an indirect effect of $\beta=0.06$, $\mathrm{p}=0.03$ ) and, to a lesser extent, negatively related with perceived impact of disease $(\beta=-0.17 ; p=0.02)$. This impact, in turn, was positively related to disease activity $(\beta=0.36 ; p<0.001)$ and mitigated by 'positive' personality traits $(\beta=-0.37$; $p<0.001)$. Impact of disease had a much stronger relation with QoL than with happiness (total effect of 0.72 , of which $\beta=-0.02, p=0.04$ was an indirect effect vs $\beta=-$ $0.17 ; p=0.02$, respectively). Happiness mitigated the negative effect of disease impact upon QoL $(\beta=0.13 ; p=0.01)$. Moreover, disease activity had a negative indirect effect of $-0.26(p=0.003)$ on $Q o L$ and also a negative indirect effect of $\beta=-$ $0.06(p=0.04)$ on happiness.

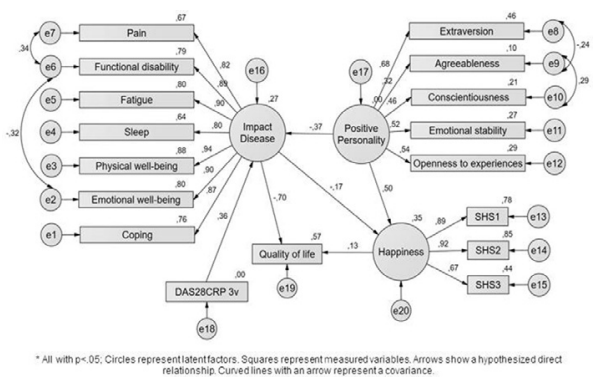

Abstract OP0160-HPR - Figure 1. Estimated standerdised direct effect for the proposed model.
Conclusions: Optimisation of QoL and happiness of people with RA requires not only effective control of the disease process but also improvement of the disease impact domains. Personality, and its effects upon the patient's perception and experience of life, seems to play a pivotal mediating role in these relations and should deserve paramount attention if happiness and enjoyment of life is taken as the ultimate goal of health care.

Disclosure of Interest: None declared

DOI: 10.1136/annrheumdis-2018-eular.1455

\section{OP0161-HPR THE DEVELOPMENT OF SELF-MANAGEMENT TRAINING FOR INNOVATIVE DEPARTMENTS (STRIDE): A SKILLS- TRAINING PROGRAMME FOR RHEUMATOLOGY TEAMS TO ENHANCE SUPPORT FOR SELF-MANAGEMENT}

S. Halls ${ }^{1}$, E. Dures ${ }^{1}$, S. Hewlett ${ }^{1}$, S. Ryan ${ }^{2}$, R. Jenkins ${ }^{3}$, J. van Tonder ${ }^{3} .{ }^{1}$ Nursing and Midwifery, The University of the West of England, Bristol; ${ }^{2}$ Staffordshire and Stoke on Trent Partnership NHS Trust, Haywood Hospital, Stoke on Trent; ${ }^{3}$ Rheumatology Department, Bristol Royal Infirmary, Bristol, UK

Background: Integrating self-management support into clinical practice is a key NHS, UK, EU and USA policy. ${ }^{1-3}$ A survey with $>1000$ UK patients with inflammatory arthritis showed that many would like support to manage the impact of symptoms $(82 \%)$, emotions (57\%), and depression (34\%). ${ }^{4}$ However, rheumatology teams rated the support they offer as inadequate, and one of the main reasons is the lack of appropriate skills-training for healthcare professionals. ${ }^{5}$ Evidence suggests that self-management support provision works best when it is condition-specific and has team and organisational support. ${ }^{6} 7$

Objectives: To develop a skills-training programme for rheumatology teams to enhance support for self-management provision through enhancing current skills and facilitating self-management strategy development.

Methods: The steering team comprised clinicians, academics with expertise in self-management and HP education, and two patient partners. To inform the practicalities and structure of the programme and optimise feasibility and acceptability, qualitative telephone interviews were performed with 11 rheumatology healthcare professionals, focusing on barriers and enablers of providing self-management support, and how to optimise buy-in.

Results: Interviews identified key considerations and practical recommendations for programme development including: the challenge vs value of involving the whole team; providing opportunities to practice skills; focusing on how to implement skills in clinical practice; and using peer support and prompts to develop and maintain skills. The Self-Management Training for Innovative Departments (STRIDE) programme was therefore formulated to comprise two 3 hour training sessions to whole teams at local departments, approximately 4-6 weeks apart (6 CPD hours). It contains modules on self-management, motivational interviewing, collaborative care and agenda setting, communication and acceptance, and signposting (eg for psychological or peer support) and the opportunity for teams to review their own practice and patient pathways. Delivery involves presentation of the evidence base, group discussion, 4 patient videos, skills demonstrations, and practice and feedback opportunities, facilitated by two expert trainers. Teams are provided with a localised support package after each session, which includes individual behavioural/attitudinal issues and team care provision points discussed during training and raised as potential areas to take forward.

Conclusions: Interviews highlighted the challenges in developing team training and enabled the development of a practical, brief training programme. The STRIDE programme is currently being piloted and evaluated with five rheumatology teams across England. Evaluation involves assessment of healthcare profes sional skills, knowledge and confidence (pre and post training questionnaires); healthcare professional experiences of training (qualitative interviews); and a patient-based evaluation (pre and post training questionnaires).

\section{REFERENCES:}

[1] NICE (2009)

[2] Forestier, et al. (2009)

[3] ACR (2002).

[4] Dures, et al. (2014a)

[5] Dures, et al. (2014b).

[6] National Voices (2014)

[7] Taylor, et al. (2014).

Acknowledgements: To the healthcare professionals who participated in interviews.

Disclosure of Interest: None declared

DOI: 10.1136/annrheumdis-2018-eular.2485 